Georgian Mathematical Journal

Volume 13 (2006), Number 2, 297-306

\title{
AN INVERSE RESULT IN SIMULTANEOUS APPROXIMATION BY MODIFIED BETA OPERATORS
}

\author{
PRERNA MAHESHWARI \\ Dedicated to Dr. Vijay Gupta, NSIT, New Delhi, India
}

\begin{abstract}
In this paper we study the modified Beta operators. We extend the result of [4] and obtain an inverse result for the linear combination of these modified Beta operators in simultaneous approximation.
\end{abstract}

2000 Mathematics Subject Classification: 41A25, 41A30.

Key words and phrases: Linear combinations, Peetre's $K$-functional, order of approximation, modulus of continuity.

\section{INTRODUCTION}

Let $f$ be a function defined on $[0, \infty)$. The modified Beta operators introduced by Gupta and Ahmad [4] are defined by

$$
B_{n}(f, x)=\frac{n-1}{n} \sum_{\nu=0}^{\infty} b_{n, \nu}(x) \int_{0}^{\infty} p_{n, \nu}(t) f(t) d t, \quad x \in[0, \infty),
$$

where

$$
b_{n, \nu}=\frac{1}{B(\nu+1, n)} \frac{x^{\nu}}{(1+x)^{n+\nu+1}}, \quad p_{n, \nu}(t)=\left(\begin{array}{c}
n+\nu+1 \\
\nu
\end{array}\right) \frac{t^{\nu}}{(1+t)^{n+\nu}} .
$$

Let $C_{\gamma}[0, \infty)=\left\{f \in[0, \infty):|f(t)| \leq M t^{\gamma}\right.$ for some $\gamma>0$ and some constant $M>0\}$. It is easily observed that for $n>\gamma$ this class of the operators $B_{n}(f, x)$ is well defined. We define the norm $\|\cdot\|_{\gamma}$ on $C_{\gamma}[0, \infty)$ by $\|f\|_{\gamma}=\sup _{0 \leq t<\infty}|f(t)| t^{-\gamma}$.

The order of approximation for these operators (1.1) is at best $O\left(n^{-1}\right)$. To improve the order of approximation, we consider the linear combination of these operators (1.1). For arbitrary but fixed distinct positive integers $d_{0}, d_{1}, \ldots, d_{k}$, the linear combination $B_{n}(f, k, x)$ of $B_{d_{j} n}(f, x), j=0,1, \ldots, n$, is defined by

$$
B_{n}(f, k, x)=\sum_{j=0}^{k} C(j, k) B_{d_{j} n}(f, x),
$$

where

$$
C(j, k)=\prod_{\substack{i=0 \\ i \neq j}} \frac{d_{j}}{d_{j}-d_{i}}, \quad k \neq 0 \quad \text { and } \quad C(0,0)=1 .
$$

In [4] the authors obtained a Voronvskaja type asymptotic formula and an error estimate in simultaneous approximation. Recently, Maheshwari and Gupta 
[5] have extended the result of [4] and obtained direct theorems for the linear combination $B_{n}(f, k, x)$ in terms of a higher order modulus of continuity. In this context we mention the recent work of V. Gupta (see, e.g., [2], [3]), who studied another type of discretely defined summation integral type operators and estimated local direct results in ordinary and simultaneous approximation. In the present paper the results of [4] and [5] are extended. It should be noted that there were many typing errors in [5], which are corrected in the present paper. Here we obtain an inverse result in simultaneous approximation by the linear combination $B_{n}(f, k, x)$.

We may rewrite operators (1.1) as

$$
B_{n}(f, x)=\int_{0}^{\infty} P_{n}(x, t) f(t) d t,
$$

where the kernel $P_{n}(x, t)$ is given by

$$
P_{n}(x, t)=\frac{n-1}{n} \sum_{\nu=0}^{\infty} b_{n, \nu}(x) p_{n, \nu}(t) .
$$

\section{Auxiliary Results}

This section contains the basic results and definitions needed to prove our main theorem.

Throughout the paper it is assumed that $0<a_{1}<a_{2}<b_{2}<b_{1}<\infty$.

Definition 1. A continuous function $f$ on the interval $[a, b]$ is said to belong to the generalized Zygmund class $Z_{\alpha}(k, a, b), 0<\alpha<2, k \in N$, if there exists a constant $C$ such that

$$
\omega_{2 k}(f, \delta, a, b) \leq C \delta^{\alpha k}, \quad \delta>0,
$$

where $\omega_{2 k}(f, \delta, a, b)$ denotes the modulus of continuity of $2 k$-th order of $f$ on the interval $[a, b]$. In particular we denote by $Z_{\alpha}^{*}$ the class $Z_{\alpha}(1, a, b)$.

Definition 2. Let $C_{0}$ denote the class of continuous functions on the interval $[0, \infty)$ having a compact support, and $C_{0}^{k}$ be a subset of $C_{0}$ of $k$ times continuously differentiable functions. Suppose $m \in N_{0} \equiv N \cup\{0\},\left[a^{\prime}, b^{\prime}\right] \subset$ $(a, b),[a, b] \subset(0, \infty)$ and for a fixed $k \in N_{0}$, let $G^{(m)}=\left\{g: g \in C_{0}^{2 k+m+2}\right.$, $\left.\operatorname{supp}(g) \subset\left[a^{\prime}, b^{\prime}\right]\right\}$. For $m$ times continuously differentiable functions $f$ with $\operatorname{supp}(f) \subset\left[a^{\prime}, b^{\prime}\right]$, the Peetre's $K$-functional is defined as

$$
\begin{gathered}
K_{m}(\xi, f) \\
=\inf _{g \in G^{(m)}}\left[\left\|f^{(m)}-g^{(m)}\right\|_{C\left[a^{\prime}, b^{\prime}\right]}+\xi\left\{\left\|g^{(m)}\right\|_{C\left[a^{\prime}, b^{\prime}\right]}+\left\|g^{(2 k+m+2)}\right\|_{C\left[a^{\prime}, b^{\prime}\right]}\right\}\right],
\end{gathered}
$$

where $0<\xi<1$. For $0<\alpha<2$, we define by $C_{0}^{m}\left(a, k, a^{\prime}, b^{\prime}\right)$ the class of $m$ times continuously differentiable functions $f$ with $\operatorname{supp}(f) \subset\left[a^{\prime}, b^{\prime}\right]$ satisfying the condition

$$
\sup _{0<\xi \leq 1} \xi^{-\alpha / 2} K_{m}(\xi, f)<M \quad \text { for some constant } \quad M>0 .
$$


In the following two lemmas $[\beta]$ denotes the integer part of $\beta$ and a constant in $O(\cdot)$ depends on $x$.

Lemma $2.1([4])$. For $m \in N_{0}$, the polynomial $U_{n, m}(x)=\frac{1}{n} \sum_{\nu=0}^{\infty} b_{n, \nu}(x)\left(\frac{\nu}{n+1}-\right.$ $x)^{m}$ satisfies the following recurrence relation:

$$
(n+1) U_{n, m+1}(x)=x(1+x)\left[U_{n, m}^{\prime}(x)+m U_{n, m+1}(x)\right]
$$

which implies that

(i) $U_{n, m}(x)$ is a polynomial of $x$ of degree $\leq m$;

(ii) $U_{n, m}(x)=O\left(n^{-[(m+1) / 2]}\right)$.

Lemma $2.2([4])$. For $m \in N_{0}, n \in N, x \in[0, \infty)$ the m-th order moment is defined by

$$
T_{n, m}(x)=\frac{n-1}{n} \sum_{\nu=0}^{\infty} b_{n, \nu}(x) \int_{0}^{\infty} p_{n, \nu}(t)(t-x)^{m} d t,
$$

then $T_{n, 0}=1, T_{n, 1}=\frac{3 x+1}{n-2}$ and we have the recurrence relation

$$
\begin{aligned}
(n-m-2) T_{n, m+1}(x)= & x(1+x)\left[T_{n, m}(x)+2 m T_{n, m-1}(x)\right] \\
& +[(1+2 x)(m+1)+x] T_{n, m}(x), \quad n>m+2,
\end{aligned}
$$

which for all $x \in[0, \infty)$ implies $T_{n, m}(x)=O\left(n^{[(m+1) / 2]}\right)$.

Corollary $2.3([4])$. Let $\delta$ be a positive number, then for every $n>\gamma>0$ and $x \in[0, \infty)$, there exists a constant $K_{m, x}$ depending on $m$ and $x$ :

$$
\int_{|t-x|>\delta} P_{n}(x, t) t^{\gamma} d t \leq K_{m, x} n^{-m} \quad \text { for some } m \in N .
$$

Lemma 2.4. There exist polynomials $\phi_{i, j, r}(x)$ independent of $n$ and $\nu$ such that

$$
[x(1+x)]^{r} \frac{d^{r}}{d x^{r}}\left(b_{n, \nu}(x)\right)=\sum_{\substack{2 i+j \leq r, i, j \geq 0}}(n+1)^{i}[\nu-(n+1) x]^{j} \phi_{i, j, r}(x) b_{n, \nu}(x) .
$$

Theorem $2.5([5])$. Let $f \in C_{\gamma}[0, \infty)$. If $f^{(2 k+m+2)}$ exists at a point $x \in$ $[0, \infty)$, then

$$
\lim _{n \rightarrow \infty} n^{k+1}\left\{B_{n}^{(m)}(f, k, x)-f^{(m)}(x)\right\}=\sum_{i=r}^{2 k+m+2} Q(i, k, m, x) f^{(i)}(x),
$$

where $Q(i, k, m, x)$ are certain polynomials in $x$.

In what follows $C_{1}, C_{2}, \ldots$ stand for the positive constant.

Lemma 2.6. Let $0<a<a^{\prime}<a^{\prime \prime}<b^{\prime \prime}<b^{\prime}<b<\infty$. If $f^{(m)} \in C_{0}$, $\operatorname{supp}(f) \in\left[a^{\prime \prime}, b^{\prime \prime}\right]$ and

$$
\left\|B_{n}^{(m)}(f, k, \cdot)-f^{(m)}(x)\right\|_{C[a, b]}=O\left(n^{-\alpha(k+1) / 2}\right),
$$


then

$$
K_{m}(\eta, f)=C_{1}\left\{n^{-\alpha(k+1) / 2}+n^{k+1} \eta K_{m}\left(n^{-(k+1)}, f\right)\right\} .
$$

As a consequence, $K_{m}(\eta, f) \leq C_{2} \eta^{\alpha / 2}$, i.e., $f \in C_{0}^{m}\left(\alpha, k+1, a^{\prime}, b^{\prime}\right)$.

Proof. To prove (2.2), it is sufficient to show that

$$
K_{m}(\eta, f)=C_{1}\left\{n^{-\alpha(k+1) / 2}+n^{k+1} \eta K_{m}\left(n^{-(k+1)}, f\right)\right\} \text { for sufficiently large } n \text {. }
$$

Now as $\operatorname{supp}(f) \subset\left[a^{\prime \prime}, b^{\prime \prime}\right]$ in view of Theorem 2.5 there exists a function $g^{i} \in$ $G^{(m)}$ such that for $i=m$ and $i=2 k+m+2$

$$
\begin{gathered}
\left\|B_{n}^{(i)}(f, k, \cdot)-g^{(i)}\right\|_{C[a, b]} \leq C_{2} n^{-(k+1)}, \\
K_{m}(\eta, f) \leq 3 C_{3} n^{-1}+\left\|B_{n}^{(m)}(f, k, \cdot)-f^{(m)}\right\|_{C\left[a^{\prime}, b^{\prime}\right]} \\
+\eta\left\{\left\|B_{n}^{(m)}(f, k, \cdot)\right\|_{C\left[a^{\prime}, b^{\prime}\right]}+\left\|B_{n}^{(2 k+m+2)}(f, k, \cdot)\right\|_{C\left[a^{\prime}, b^{\prime}\right]}\right\} .
\end{gathered}
$$

Thus it suffices to show that there exists a constant $C_{4}$ such that for each $h \in G^{(m)}$

$$
\begin{gathered}
\left\|B_{n}^{(2 k+m+2)}(f, k, \cdot)\right\|_{C\left[a^{\prime}, b^{\prime}\right]} \\
\leq C_{4} n^{k+1}\left\{\left\|f^{(m)}-h^{(m)}\right\|_{C\left[a^{\prime}, b^{\prime}\right]}+n^{-(k+1)}\left\|h^{(2 k+m+2)}\right\|_{C\left[a^{\prime}, b^{\prime}\right]}\right\} .
\end{gathered}
$$

Again $B_{n}^{(2 k+m+2)}(f, k, \cdot)$ satisfies the linearity property

$$
\begin{gathered}
\left\|B_{n}^{(2 k+m+2)}(f, k, \cdot)\right\|_{C\left[a^{\prime}, b^{\prime}\right]} \\
\leq\left\|B_{n}^{(2 k+m+2)}(f-h, k, \cdot)\right\|_{C\left[a^{\prime}, b^{\prime}\right]}+\left\|B_{n}^{(2 k+m+2)}(h, k, \cdot)\right\|_{C\left[a^{\prime}, b^{\prime}\right]} .
\end{gathered}
$$

Using Lemma 2.4, we have

$$
\begin{array}{r}
\int_{0}^{\infty}\left|\frac{\partial^{2 k+m+2}}{\partial x^{2 k+m+2}} P_{n}(x, t)\right| d t \leq \sum_{\substack{2 i+j<2 k+m+2 \\
i, j \geq 0}} \frac{n-1}{n} \sum_{\nu=1}^{\infty}(n+1)^{i}|\nu-(n+1) x|^{j} \\
\times \frac{\left|\phi_{i, j, 2 k+m+2}(x)\right|}{\{x(1+x)\}^{2 k+m+2}} b_{n, \nu}(x) \int_{0}^{\infty} p_{n, \nu}(t) d t .
\end{array}
$$

Hence, by the Cauchy-Schwarz inequality, Lemma 2.1 and the fact $\int_{0}^{\infty} p_{n, \nu}(t) d t=$ $\frac{1}{n-1}$, we obtain

$$
\left\|B_{n}^{(2 k+m+2)}(f-h, k, \cdot)\right\|_{C\left[a^{\prime}, b^{\prime}\right]} \leq C_{5} n\left\|f^{(m)}-g^{(m)}\right\|_{C\left[a^{\prime}, b^{\prime}\right]},
$$

where the constant $C_{5}$ is independent of $f$ and $g$.

Now, by Taylor's expansion, we have

$$
h(t)=\sum_{i=0}^{2 k+m+1} \frac{h^{(i)}(t)}{i !}(t-x)^{i}+\frac{h^{(2 k+m+2)}(\xi)}{(2 k+m+2) !}(t-x)^{2 k+m+2},
$$


where $\xi$ lies between $t$ and $x$. Using (2.6), we have

$$
\begin{aligned}
\| \frac{\partial^{2 k+m+2}}{\partial x^{2 k+m+2}} B_{n}(g, k, \cdot) & \left\|_{C\left[a^{\prime}, b^{\prime}\right]} \leq \sum_{j=0}^{k} \frac{|C(j, k)|}{(2 k+m+2) !}\right\| g^{(2 k+m+2)} \|_{C\left[a^{\prime}, b^{\prime}\right]} \\
& \times\left\|\int_{0}^{\infty} \frac{\partial^{2 k+m+2}}{\partial x^{2 k+m+2}} B_{d_{j} n}(x, t)(t-x)^{2 k+m+2} d t\right\|_{C\left[a^{\prime}, b^{\prime}\right]} .
\end{aligned}
$$

We shall now calculate the term given in the second norm on the right-hand side. It is sufficient to consider the expression without the linear combination. Using Lemma 2.4 and the Cauchy-Schwarz inequality we have

$$
\begin{aligned}
I & =\int_{0}^{\infty}\left|\frac{\partial^{2 k+m+2}}{\partial x^{2 k+m+2}} P_{n}(x, t)\right| d t \\
& \leq \frac{n-1}{n} \sum_{\substack{2 i+s<2 k+m+2 \\
i, s \geq 0}} \sum_{r=0}^{\infty}(n+1)^{i}|\nu-(n+1) x|^{s} \frac{\left|\phi_{i, s, 2 k+m+2}(x)\right|}{\{x(1+x)\}^{2 k+m+2}} b_{n, \nu}(x) \\
& \times \int_{0}^{\infty} p_{n, \nu}(t)(t-x)^{2 k+m+2} d t .
\end{aligned}
$$

Next, using Lemma 2.1 and Lemma 2.2, we have

$$
\begin{aligned}
I \leq & \frac{n-1}{n} \sum_{\substack{2 i+s<2 k+m+2 \\
i, s \geq 0}}(n+1)^{i} \frac{\left|\phi_{i, s, 2 k+m+2}(x)\right|}{\{x(1+x)\}^{2 k+m+2}} b_{n, \nu}(x) \\
& \times\left(\frac{1}{n} \sum_{r=0}^{\infty} b_{n, \nu}(x)(\nu-(n+1) x)^{2 s}\right)^{1 / 2} \\
& \times\left(\sum_{r=0}^{\infty} b_{n . \nu}(x) \int_{0}^{\infty} p_{n, \nu}(t)(t-x)^{2 k+2 m+4} d t\right)^{1 / 2}\left(\int_{0}^{\infty} p_{n, \nu}(t) d t\right)^{1 / 2} \\
= & \sum_{\substack{2 i+s<2 k+m+2 \\
i, s \geq 0}}(n+1)^{i} \frac{\left|\phi_{i, s, 2 k+m+2}(x)\right|}{\{x(1+x)\}^{2 k+m+2}} O\left(n^{s / 2}\right) O\left(n^{-(k+m) / 2+1}\right), \\
I= & \sum_{\substack{2 i+s<2 k+m+2 \\
i, s \geq 0}}(n+1)^{i} \frac{\left|\phi_{i, s, 2 k+m+2}(x)\right|}{\{x(1+x)\}^{2 k+m+2}} O\left(n^{(2 i+s) / 2}\right) O\left(n^{-(2 k+m+2) / 2}\right) .
\end{aligned}
$$

Hence by using (2.7) and the above estimate we have

$$
\left\|B_{n}^{(2 k+m+2)}(h, k, \cdot)\right\|_{C\left[a^{\prime}, b^{\prime}\right]} \leq C_{6}\left\|h^{(2 k+m+2)}\right\|_{C\left[a^{\prime}, b^{\prime}\right]} .
$$

Combining estimates (2.4), (2.5) and (2.8), the result (2.3) follows. This completes the proof of (2.2). The other consequences are standard and can be found in $[1]$. 
Lemma 2.7. Let $0<a<a^{\prime}<a^{\prime \prime}<b^{\prime \prime}<b^{\prime}<b$ and $f^{(m)} \in C_{0}$ with $\operatorname{supp}(f) \subset\left[a^{\prime \prime}, b^{\prime \prime}\right]$, then if $f \in C_{0}^{(m)}\left(\alpha, k+1, a^{\prime}, b^{\prime}\right)$, we have $f^{(m)} \in \operatorname{Liz}(\alpha, k+$ $\left.1, a^{\prime}, b^{\prime}\right)$.

Proof. Let $|\delta|<g$ and $h \in G^{m}$, then we have with $f \in C_{0}^{(m)}\left(\alpha, k+1, a^{\prime}, b^{\prime}\right)$.

$$
\begin{aligned}
\left|\Delta_{\delta}^{2 k+2} f^{(m)}(x)\right| \leq & \left|\Delta_{\delta}^{2 k+2}\left(f^{(m)}(x)-h^{(m)}(x)\right)\right|+\left|\Delta_{\delta}^{2 k+2} h^{(m)}(x)\right| \\
\leq & 2^{2 k+2}\left\|f^{(m)}-h^{(m)}\right\|_{C\left[a^{\prime}, b^{\prime}\right]} \\
& +\delta^{2 k+2}\left\|g^{(2 k+m+2)}\right\|_{C\left[a^{\prime}, b^{\prime}\right]}+\left\|h^{(2 k+m+2)}\right\|_{C\left[a^{\prime}, b^{\prime}\right]} \\
\leq & C_{7} 2^{2 k+2} K_{\infty}\left(\delta^{2 k+2}, f\right) \leq C_{8} 2^{2 k+2} \delta^{\alpha(k+1)} .
\end{aligned}
$$

It follows that $f^{(m)} \in Z_{\alpha}\left(k+1, a^{\prime}, b^{\prime}\right)$.

Theorem $2.8([5])$. Let $f^{(m)} \in C_{\gamma}[0, \infty)$ and $0<a<a^{\prime}<b^{\prime}<b<\infty$, then for $n$ sufficiently large,

$\left\|B_{n}^{(m)}(f, k, \cdot)-f^{(m)}\right\|_{C\left[a^{\prime}, b^{\prime}\right]}=\max \left\{C_{9} \omega_{2 k+2}\left(f^{(m)}, n^{-1 / 2}, a, b\right), C_{10} n^{-(k+1)}\|f\|_{\gamma}\right\}$, where $C_{9}=C_{9}(k, m)$ and $C_{10}=C_{10}(k, m, f)$.

\section{The Main Result}

In this section, we shall prove the following inverse result.

Theorem 3.1. If $0<\alpha<2,0<a_{1}<a_{2}<b_{2}<b_{1}<\infty$ and $f \in C_{\gamma}[0, \infty)$, then for the following statements the implication (i) $\Rightarrow$ (ii) is true:

(i) $f^{(m)}$ exist on the interval $\left[a_{1}, b_{1}\right]$ and

$$
\left\|B_{n}^{(m)}(f, k, \cdot)-f^{(m)}\right\|_{C\left[a_{1}, b_{1}\right]}=O\left(n^{-\alpha(k+1) / 2}\right) ;
$$

(ii) $f^{(m)} \in Z_{\alpha}\left(k+1, a_{2}, b_{2}\right)$.

Proof. We shall prove this theorem by the principle of mathematical induction. Assuming (i), put $\tau=\alpha(k+1)$ and first consider the case $0<\tau \leq 1$. Let us choose $a^{\prime}, a^{\prime \prime}, b^{\prime}, b^{\prime \prime}$ in such a way that $a_{1}<a^{\prime}<a^{\prime \prime}<a_{2}<b_{2}<b^{\prime \prime}<b^{\prime}<b_{1}$. Also suppose $g \in C_{0}^{\infty}$ with $\operatorname{supp}(g) \subset\left[a^{\prime \prime}, b^{\prime \prime}\right]$ and $g(x)=1$ on $\left[a_{2}, b_{2}\right]$ for $x \in\left[a^{\prime}, b^{\prime}\right]$ with $D=\frac{d}{d x}$. We have

$$
\begin{aligned}
B_{n}^{(m)} & (f h, k, x)-(f h)^{(m)}(x)=D^{m}\left(B_{n}((f h)(t)-(f h)(x), k, x)\right) \\
& =D^{m}\left(B_{n}(f(t)(h(t)-h(x)), k, x)\right)+D^{m}\left(B_{n}(h(x)(f(t)-f(x)), k, x)\right) \\
& =J_{1}+J_{2}, \quad \text { say. }
\end{aligned}
$$

To estimate $J_{1}$, by the Leibniz Theorem, we have

$$
\begin{aligned}
J_{1} & =\sum_{j=0}^{k} C(j, k) \frac{\partial^{m}}{\partial x^{m}} \int_{0}^{\infty} P_{d_{j} n}(x, t) f(t)(h(t)-h(x)) d t \\
& =\sum_{j=0}^{k} C(j, k) \sum_{i=1}^{m}\left(\begin{array}{c}
m \\
i
\end{array}\right) \int_{0}^{\infty} P_{d_{j} n}^{i}(x, t) \frac{\partial^{m-i}}{\partial x^{m-i}}[f(t)(h(t)-h(x))] d t
\end{aligned}
$$




$$
\begin{aligned}
= & -\sum_{i=1}^{m-1}\left(\begin{array}{c}
m \\
i
\end{array}\right) h^{(m-i)}(x) B_{n}^{(i)}(f, k, x) \\
& +\sum_{j=0}^{k} C(j, k) \int_{0}^{\infty} P_{d_{j} n}^{(m)}(x, t) f(t)(h(t)-h(x)) d t \\
= & J_{3}+J_{4}, \quad \text { say. }
\end{aligned}
$$

Now, using Theorem 2.8, we obtain

$$
J_{3}=-\sum_{i=1}^{m-1}\left(\begin{array}{c}
m \\
i
\end{array}\right) h^{(m-i)}(x) f^{(i)}(x)+O\left(n^{-\tau / 2}\right), \quad \text { uniformly in } x \in\left[a^{\prime}, b^{\prime}\right] .
$$

Using Theorem 2.5, the Cauchy-Schwarz inequality, Taylor's expansion of $f$ and $h$ and Lemma 2.2, we get

$$
\begin{aligned}
J_{4} & =\sum_{i=1}^{m-1} \frac{h^{(i)}(x) f^{(m-1)}(x)}{i !(m-i) !} m !+O\left(n^{-\tau / 2}\right) \\
& =\sum_{i=1}^{m-1}\left(\begin{array}{c}
m \\
i
\end{array}\right) h^{(i)}(x) f^{(m-i)}(x)+O\left(n^{-\tau / 2}\right)
\end{aligned}
$$

uniformly in $x \in\left[a^{\prime}, b^{\prime}\right]$ by Corollary 2.3.

Finally, applying the Leibniz theorem, we obtain

$$
\begin{aligned}
J_{2} & =\sum_{j=0}^{k} C(j, k) \sum_{i=0}^{m}\left(\begin{array}{c}
m \\
i
\end{array}\right) \int_{0}^{\infty} P_{d_{j} n}^{i}(x, t) \frac{\partial^{m-i}}{\partial x^{m-i}}[h(t)(f(t)-f(x))] d t \\
& =\sum_{i=0}^{k}\left(\begin{array}{c}
m \\
i
\end{array}\right) h^{(m-i)}(x) B_{n}^{(i)}(f, k, x)-(f h)^{(m)}(x) \\
& =\sum_{i=0}^{k}\left(\begin{array}{c}
m \\
i
\end{array}\right) h^{(m-i)}(x) f^{(i)}(x)-(f h)^{(m)}(x)+O\left(n^{-\tau / 2}\right) \\
& =O\left(n^{-\tau / 2}\right) \quad \text { uniformly in } x \in\left[a^{\prime}, b^{\prime}\right] .
\end{aligned}
$$

Combining these estimates, we have

$$
\left\|B_{n}^{(m)}(f h, k, \cdot)-(f h)^{(m)}\right\|_{C\left[a^{\prime}, b^{\prime}\right]}=O\left(n^{-\tau / 2}\right) .
$$

Hence by Lemma 2.6 and Lemma 2.7, we have $(f h)^{(m)} \in Z_{\alpha}\left(k+1, a^{\prime}, b^{\prime}\right)$. Since $h(x)=1$ on $\left[a_{2}, b_{2}\right]$, it follows that $f^{(m)} \in Z_{\alpha}\left(k+1, a_{2}, b_{2}\right)$, This proves (i) $\Rightarrow$ (ii) for the case $0<\tau \leq 1$. Now to prove the implication for $0<\tau<2 k+2$ it is sufficient to assume it for $\tau \in\left(p^{\prime}-1, p^{\prime}\right)$ and prove it for $\tau \in\left(p^{\prime}, p^{\prime}+1\right)$ $\left(p^{\prime}=1,2, \ldots, 2 k+1\right)$. Since the result holds for $\tau \in\left(p^{\prime}-1, p^{\prime}\right), f^{\left(p^{\prime}+m+1\right)}$ exists and belongs to the class $Z_{\alpha}\left(1-\delta, a^{*}, b^{*}\right)$ for any $\delta>0$ and for any interval $\left(a^{*}, b^{*}\right) \subset\left(a_{1}, b_{1}\right)$. Let $a_{i}^{*}, b_{i}^{*}, i=1,2$, be such that $\left(a_{2}, b_{2}\right) \subset\left(a_{2}^{*}, b_{2}^{*}\right) \subset\left(a_{1}^{*}, b_{1}^{*}\right)$. 
Let $h \in C_{0}^{\infty}$ be such that $h(x)=1$ on $\left[a_{2}, b_{2}\right]$ and $\operatorname{supp}(h) \subset\left[a_{2}^{*}, b_{2}^{*}\right]$. Then $\zeta_{2}(t)$ denotes the characteristic function of the interval $\left[a_{1}^{*}, b_{1}^{*}\right]$, we have

$$
\begin{aligned}
\left\|B_{n}^{(m)}(f h, k, \cdot)-(f h)^{(m)}\right\|_{C\left[a_{2}^{*}, b_{2}^{*}\right]} \leq & \| D^{m}\left[B_{n}(h(x)(f(t)-f(x), k, \cdot)]\|\|_{C\left[a_{2}^{*}, b_{2}^{*}\right]}\right. \\
& +\| D^{m}\left[B_{n}(f(x)(h(t)-h(x), k, \cdot)] \|_{C\left[a_{2}^{*}, b_{2}^{*}\right]}\right. \\
= & I_{1}+I_{2} \quad(\text { say }) .
\end{aligned}
$$

To estimate $I_{1}$, by Theorem 2.5, we have

$$
\begin{aligned}
I_{1} & \leq\left\|D^{m}\left[B_{n}(h(x) f(t), k, \cdot)\right]-(f h)^{(m)}\right\|_{C\left[a_{2}^{*}, b_{2}^{*}\right]} \\
& =\left\|\sum_{i=0}^{\infty}\left(\begin{array}{c}
m \\
i
\end{array}\right) h^{(m-i)} B_{n}^{(i)}(f, k, \cdot)-(f h)^{(m)}\right\|_{C\left[a_{2}^{*}, b_{2}^{*}\right]} \\
& =\left\|\sum_{i=0}^{\infty}\left(\begin{array}{c}
m \\
i
\end{array}\right) h^{(m-i)} f^{(i)}-(f h)^{(m)}\right\|_{C\left[a_{2}^{*}, b_{2}^{*}\right]}+O\left(n^{-\tau / 2}\right)=O\left(n^{-\tau / 2}\right) .
\end{aligned}
$$

Also by Leibniz Theorem and Theorem 2.5, we have

$$
\begin{aligned}
I_{2} & \left.=\|-\sum_{i=0}^{\infty}\left(\begin{array}{c}
m \\
i
\end{array}\right) h^{(m-i)} B_{n}^{(i)}(f, k, \cdot)+B_{n}^{(m)}\left(f(t)(h(t)-h(\cdot)) \zeta_{2}(t), k, \cdot\right)\right\}_{C\left[a_{2}^{*}, b_{2}^{*}\right]} \\
& =\left\|I_{3}+I_{4}\right\|_{C\left[a_{2}^{*}, b_{2}^{*}\right]}+O\left(n^{-(k+1)}\right), \quad \text { say. }
\end{aligned}
$$

Then by Theorem 2.5, we get

$$
I_{3}=-\sum_{i=0}^{m-1}\left(\begin{array}{c}
m \\
i
\end{array}\right) h^{(m-i)}(x) f^{(i)}(x)+O\left(n^{-\tau / 2}\right), \quad \text { uniformly in } x \in\left[a_{2}^{*}, b_{2}^{*}\right] .
$$

Applying Taylor's expansion of $f$, we have

$$
\begin{aligned}
I_{4} & =\sum_{j=0}^{k} C(j, k) \int_{0}^{\infty} P_{d_{j} n}^{(m)}(x, t)\left[f(t)(h(t)-h(x)) \zeta_{2}(t)\right] d t \\
= & \sum_{j=0}^{k} C(j, k) \sum_{i=0}^{p^{\prime}+m+1} \frac{f^{(i)}(x)}{i !} \int_{0}^{\infty} P_{d_{j} n}^{(m)}(x, t)(t-x)^{i}(h(t)-h(x)) \zeta_{2}(t) d t \\
= & \sum_{j=0}^{k} C(j, k) \int_{0}^{\infty} P_{d_{j} n}^{(m)}(x, t)\left(\frac{f^{\left(p^{\prime}+m-1\right)}(\eta)-f^{\left(p^{\prime}+m-1\right)}(x)}{\left(p^{\prime}+m-1\right) !}\right) \\
& \times(t-x)^{\left(p^{\prime}+m-1\right)}(h(t)-h(x)) \zeta_{2}(t) d t
\end{aligned}
$$

( $\eta$ lying between $t$ and $x$ )

$$
=I_{5}+I_{6}, \quad \text { say. }
$$

Applying Theorem 2.5, we get

$$
I_{5}=\sum_{j=0}^{k} C(j, k) \sum_{i=0}^{p^{\prime}+m+1} \frac{f^{(i)}(x)}{i !} \int_{0}^{\infty} P_{d_{j} n}^{(m)}(x, t)(t-x)^{i}(h(t)-h(x)) d t+O\left(n^{-(k+1)}\right)
$$




$$
=I_{7}+O\left(n^{-(k+1)}\right), \text { say. }
$$

Since $h \in C_{0}^{\infty}$, we can write

$$
\begin{aligned}
I_{7}= & \sum_{j=0}^{k} C(j, k) \sum_{i=0}^{p^{\prime}+m+1} \frac{f^{(i)}(x)}{i !} \sum_{r=0}^{p^{\prime}+m+1} \frac{h^{(r)}(x)}{r !} \int_{0}^{\infty} P_{d_{j} n}^{(m)}(x, t)(t-x)^{i+r} d t \\
& +\sum_{j=0}^{k} C(j, k) \sum_{i=0}^{p^{\prime}+m+1} \frac{f^{(i)}(x)}{i !} \int_{0}^{\infty} P_{d_{j} n}^{(m)}(x, t) \varepsilon(t, x)(t-x)^{i+p^{\prime}+m+1} d t \\
& \quad \text { (where } \varepsilon(t, x) \rightarrow 0 \text { as } t \rightarrow x) \\
= & I_{8}+I_{9}, \quad \text { say. }
\end{aligned}
$$

Next, by Theorem 2.5 we get

$$
I_{8}=\sum_{r=1}^{m} \frac{h^{(r)}}{r !} \frac{f^{(m-r)}(x)}{(m-r) !} m !+O\left(n^{-(k+1)}\right)=\sum_{r=0}^{m}\left(\begin{array}{c}
m \\
i
\end{array}\right) h^{(r)}(x)+O\left(n^{-(k+1)}\right) .
$$

Also, $I_{9}=O\left(n^{-\tau / 2}\right)$ uniformly in $x \in\left[a_{2}^{*}, b_{2}^{*}\right]$.

Finally, by the mean value theorem,

$$
\begin{aligned}
& \left\|I_{6}\right\|_{C\left[a_{2}^{*}, b_{2}^{*}\right]} \leq \sum_{j=0}^{k}|C(j, k)| \\
& \times\left\|\int_{0}^{\infty} P_{d_{j} n}^{(m)}(x, t) \frac{f^{\left(p^{\prime}+m-1\right)}(\eta)-f^{\left(p^{\prime}+m-1\right)}(x)}{\left(p^{\prime}+m-1\right) !}\left|h^{\prime}(\xi)\right||t-x|^{p^{\prime}+m} \zeta_{2}(t) d t\right\|_{C\left[a_{2}^{*}, b_{2}^{*}\right]},
\end{aligned}
$$

where $\xi$ and $\eta$ lie between $t$ and $x$.

Using Lemma 2.4, we have

$$
\begin{aligned}
\left\|I_{6}\right\|_{C\left[a_{2}^{*}, b_{2}^{*}\right]} \leq \sum_{j=0}^{k}|C(j, k)| \sum_{\substack{2 r+s \leq m \\
r, s \geq 0}}\left(d_{j} n+1\right)^{r}\left\|h^{\prime}\right\|_{C\left[a_{2}^{*}, b_{2}^{*}\right]} \\
\times \| \frac{\phi_{r, s, m}(x)}{\{x(1+x)\}^{m}} \int_{0}^{\infty} P_{d_{j} n}(x, t)\left|\nu-\left(d_{j} n+1\right) x\right|^{s} \\
\times \frac{f^{\left(p^{\prime}+m-1\right)}(\eta)-f^{\left(p^{\prime}+m-1\right)}(x)}{\left(p^{\prime}+m-1\right) !}|t-x|^{p^{\prime}+m} \zeta_{2}(t) \|_{C\left[a_{2}^{*}, b_{2}^{*}\right]} .
\end{aligned}
$$

Now using the Cauchy-Schwarz inequality, Lemma 2.1 and Lemma 2.2, we get

$$
\left\|I_{6}\right\|_{C\left[a_{2}^{*}, b_{2}^{*}\right]}=O\left(n^{-\left(p^{\prime}+1-\delta\right) / 2}\right)=O\left(n^{-\tau / 2}\right)
$$

by choosing $\delta$ such that $0 \leq \delta \leq p^{\prime}+1-\tau$.

Combining the above estimates, we get

$$
\left\|B_{n}^{(m)}(f h, k, \cdot)-(f h)^{(m)}\right\|_{C\left[a_{2}^{*}, b_{2}^{*}\right]}=O\left(n^{-\tau / 2}\right) .
$$


Since $\operatorname{supp}(f h) \subset\left[a_{2}^{*}, b_{2}^{*}\right]$, it follows from Lemma 2.5 and Lemma 2.7 that

$$
(f h)^{(m)} \in Z_{\alpha}\left(k+1, a_{2}^{*}, b_{2}^{*}\right) .
$$

Since $h(x)=1$ on $\left[a_{2}, b_{2}\right]$, we have $f^{(m)} \in Z_{\alpha}\left(k+1, a_{2}^{*}, b_{2}^{*}\right)$.

This completes the proof of Theorem 3.1.

\section{ACKNOWLEDGEMENT}

The author is extremely thankful to the referee for her/his valuable advice.

\section{REFERENCES}

1. H. Berens and G. G. Lorentz, Inverse theorems for Bernstein polynomials. Indiana Univ. Math. J. 21(1971/72), 693-708.

2. V. Gupta, Some approximation properties for modified Baskakov type operators. Georgian Math. J. 12(2005), No. 2, 217-228.

3. V. Gupta, Error estimation by mixed summation integral type operators. J. Math. Anal. Appl. 313(2006), No. 2, 632-641.

4. V. Gupta and A. Ahmad, Simultaneous approximation by modified beta operators. Istanbul niv. Fen Fak. Mat. Derg. 54(1995), 11-22 (1997).

5. P. Maheshwari and V. Gupta, An estimation on the rate of convergence for modified beta operators. Indian J. Pure Appl. Math. 34(2003), No. 6, 927-934.

6. A. F. Timan, Theory of approximation of functions of a real variable. (Translated from the Russian) International Series of Monographs in Pure and Applied Mathematics, Vol. 34. A Pergamon Press Book. The Macmillan Co., New York, 1963.

(Received 16.01.2005; revised 28.05.2006)

Author's address:

Department of Mathematics

H. R. Institute of Technology

Meerut Road, Ghaziabad-201005 (U.P.)

India

E-mail: mprerna_anand@yahoo.com 